\title{
Identificación de Hantavirus Andes en Rattus norvegicus
}

\author{
Identification of Andes Hantavirus in Rattus norvegicus

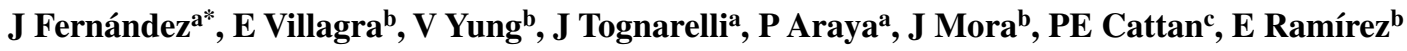 \\ a Sección Genética Molecular y bubdepto. de Virología, Instituto de Salud Pública de Chile, Marathon 1000, Santiago, Chile. \\ ${ }^{c}$ Universidad de Chile, Facultad de Ciencias Veterinarias, Avenida Santa Rosa 11735, La Pintana, Chile.
}

\begin{abstract}
SUMMARY
In Chile and Argentina Oligoryzomys longicaudatus has been identified as the main reservoir for Hantavirus Andes. The aim of this work was to report the presence of Hantavirus Andes in Rattus norvegicus, an urban rodent collected during sampling in San Diego del Cristo, Melipilla, Metropolitan Region. The existence of Hantavirus Andes in Rattus norvegicus could indicate the importance of other rodents as possible viral vectors.
\end{abstract}

Palabras clave: Hantavirus, roedores, síndrome cardiopulmonar, virus Andes.

Keywords: Hantavirus, rodents, cardiopulmonar syndrome, Andes virus.

\section{INTRODUCCION}

Hantavirus produce dos tipos de enfermedad en humanos: fiebre hemorrágica con compromiso renal (FHR) con una tasa de mortalidad de 0,1 a 15\% en Asia y Europa; y síndrome cardiopulmonar por Hantavirus (SCPH) con una tasa de mortalidad de 40\% en América. Ambas zoonosis son transmitidas al hombre al inhalar aerosoles de heces, orina y saliva de roedores infectados (Wells y col 1997, Cantoni y col 2001).

El género Hantavirus, familia Bunyaviridae, comprende a lo menos 20 diferentes clases de virus (Mertz y col 1997, Rossi y col 1999). Cada uno está asociado predominantemente a un huésped específico del área geográfica donde el virus fue caracterizado, aun cuando ocasionalmente ocurre transmisión de infecciones a otras especies de roedores (Hjelle y Yates 2001).

Roedores de la subfamilia Sigmodontinae son reservorio para distintos tipos de Hantavirus a través del continente americano. En Chile y Argentina la especie Oligoryzomys longicaudatus ha sido identificada como el principal reservorio para Hantavirus Andes (Padula y col 2000, Galeno y col 2002).

Desde que el SCPH fuera detectado por primera vez en Chile en 1995 causando un gran impacto en los sistemas de salud, su forma de presentación ha variado y desde 1997 se presenta como una endemia, con casos esporádicos y brotes estacionales. Las tasas de mortalidad más elevadas ocurrieron en los años 1997, 1998 y 2001, a partir de entonces se

Aceptado: 30.04.2008.

* Sección Genética Molecular, Departamento de Laboratorios Biomédicos Nacional y Referencia, Instituto de Salud Pública de Chile, Marathon 1000, Ñuñoa, Santiago, Chile; jfernand@ispch.cl han mantenido estables con una tasa de 0,12 por 100.000 habitantes (Sotomayor y Marincovich 2005). Estudios de Hantavirus Andes en humanos realizados en el sur de Chile (Aysén) indican valores de seroprevalencia de $2 \%$ en áreas urbanas y $13 \%$ en áreas rurales (Tager-Frey y col 2003).

Recientemente se han reportado casos de síndrome cardiopulmonar por Hantavirus asociados con virus circulante en roedores silvestres en Sudamérica, sin embargo no ha sido demostrada la presencia de Hantavirus en roedores urbanos. La asociación entre roedores urbanos infectados por Hantavirus y casos de personas infectadas por el mismo virus ha sido demostrada en Asia (Lee 1999). Este trabajo reporta la identificación de una variante Hantavirus Andes en un roedor urbano capturado en San Diego del Cristo, comuna de Melipilla, ubicada en la zona central del país.

\section{MATERIAL Y METODOS}

Roedores: A raíz de un caso de SCPH en San Diego del Cristo (figura 1), se realizó entre el 31 de octubre y el 3 de noviembre del 2000 un muestreo donde fueron capturados 40 roedores. Cada roedor fue identificado y clasificado morfológicamente por sus características fenotípicas, a cada uno de ellos se le tomó una muestra de sangre y de tejido pulmonar.

Las muestras serológicas obtenidas de los 40 roedores fueron analizadas para IgG por la técnica de ELISA con antígeno de Hantavirus Andes (Plyusnin y col 2001).

El ARN total del tejido pulmonar obtenido de los roedores fue extraído con TRIzol-LS (Invitrogene), posteriormente el ARN purificado fue resuspendido en agua tratada con dietilpirocarbonato (DEPC, SIGMA) y con 20 unidades de inhibidor de ribonucleasa (CPG ING) (López y col 1997). La síntesis de ADN complementario fue realizada 


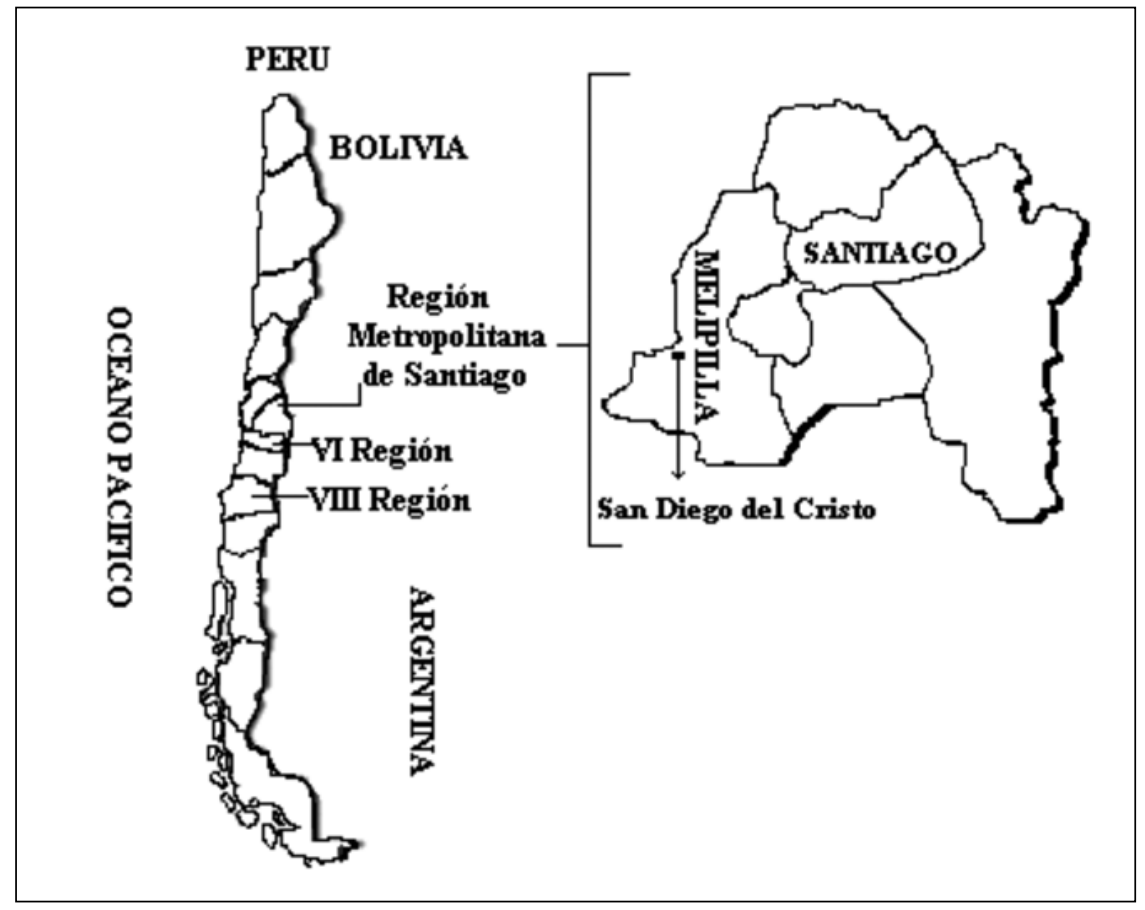

Figura 1. Area geográfica del lugar de muestreo.

Geographic trapping area of rodents.

mediante transcripción reversa (TR), en una solución que contenía: $50 \mathrm{mM}$ de Tris- $\mathrm{HCl}$ (pH 8,0), 3 mM MgCL 2,75 $\mathrm{mM} \mathrm{KCL}, 10 \mathrm{U}$ de inhibidor de ribonucleasa, $10 \mathrm{mM}$ de dithiotreitol (DTT), $200 \mathrm{ng}$ de hexamero y $50 \mathrm{U}$ de la enzima transcripasa reversa M-MLV (Invitrogen). La reacción de transcripción reversa se realizó a $37^{\circ} \mathrm{C}$ durante una hora. El ADN complementario generado se utilizó como templado para la amplificación mediante la reacción en cadena de la polimerasa (RCP), de un fragmento de 303 pares de bases correspondiente al gen que codifica para la nucleoproteína de Andes virus (López y col 1997). La reacción contenía: $20 \mathrm{mM}$ de Tris-HCL (pH 8,4), $50 \mathrm{mM}$ KCL, $1,5 \mathrm{mM}$ de $\mathrm{MgCl}_{2}, 2 \mathrm{mM}$ de cada uno de los desoxinucleótidos trifosfatos (dNTP's), $20 \mu \mathrm{M}$ de cada iniciador y 1,0 U de la enzima Taq polimerasa (Invitrogen). La amplificación fue realizada en un termociclador (modelo 2700 de Applied Biosystem) y consistió en 35 ciclos de 1 minuto a $94^{\circ} \mathrm{C}, 1$ minuto a $55^{\circ} \mathrm{C}$ y 1 minuto a $72{ }^{\circ} \mathrm{C}$. El fragmento amplificado mediante RCP fue purificado con columnas QIAquick (QIAGEN Inc.), siguiendo las instrucciones del fabricante.

El fragmento purificado fue secuenciado con la pareja de iniciadores previamente reportados (López y col 1997), usando el método automático de ABI Prism 310 (Applied Biosystem) siguiendo las instrucciones del proveedor. La secuencia obtenida fue comparada con secuencias prototipos de Hantavirus de la base de datos Genbank. El análisis filogenético fue realizado con el programa computacional MEGA 3.1, utilizando el algoritmo neighbour-joining, parsimonia y el método bootstrap con 1.000 repeticiones.

\section{RESULTADOS Y DISCUSION}

La identificación morfológica de los 40 roedores capturados demostró que 29 roedores fueron identificados como Abrothrix olivaceus, tres roedores como Oligoryzomys longicaudatus, dos fueron clasificados como Rattus rattus y seis correspondieron a Rattus norvegicus.

La determinación de $\operatorname{IgG}$ virus Andes mediante el método de ELISA en los 40 sueros de roedores capturados demostró que sólo el suero del roedor Rattus norvegicus fue positivo para anticuerpos IgG de Hantavirus Andes. El análisis del ARN total obtenido desde el tejido pulmonar del roedor Rattus norvegicus, mediante TR-RCP, permitió la detección de un fragmento de 303 pares de base correspondiente al gen que codifica para la nucleoproteína de Hantavirus Andes. El análisis de la secuencia nucleotídica demostró una similitud de un $90,1 \%$ con respecto a la secuencia nucleotídica de Hantavirus Andes (AH-1) de Argentina. El análisis de la secuencia de aminoácidos demostró un $100 \%$ similitud con respecto a la secuencia de aminoácidos Hantavirus Andes AH-1 de Argentina. Al comparar la secuencia con el aislado chileno de Hantavirus CH-7913, se obtiene un $89 \%$ de similitud con respecto a la secuencia nucleotídica y un $99 \%$ de similitud con respecto a la secuencia de aminoácidos. En cambio, al comparar la secuencia nucleotídica con otros Hantavirus (Seoul, Hantaan, Black Creek Canal, Bayou, New York/ RI-1, Sin Nombre/cc107, Sin Nombre/mnh1, Prospect Hill, Puumala) descritos en la literatura, se obtiene un índice de similitud inferior al $80 \%$. 
El análisis filogenético demuestra que la secuencia nucleotídica obtenida del roedor Rattus norvegicus está relacionada genéticamente con Hantavirus Andes que circula en Argentina y Chile (figura 2).

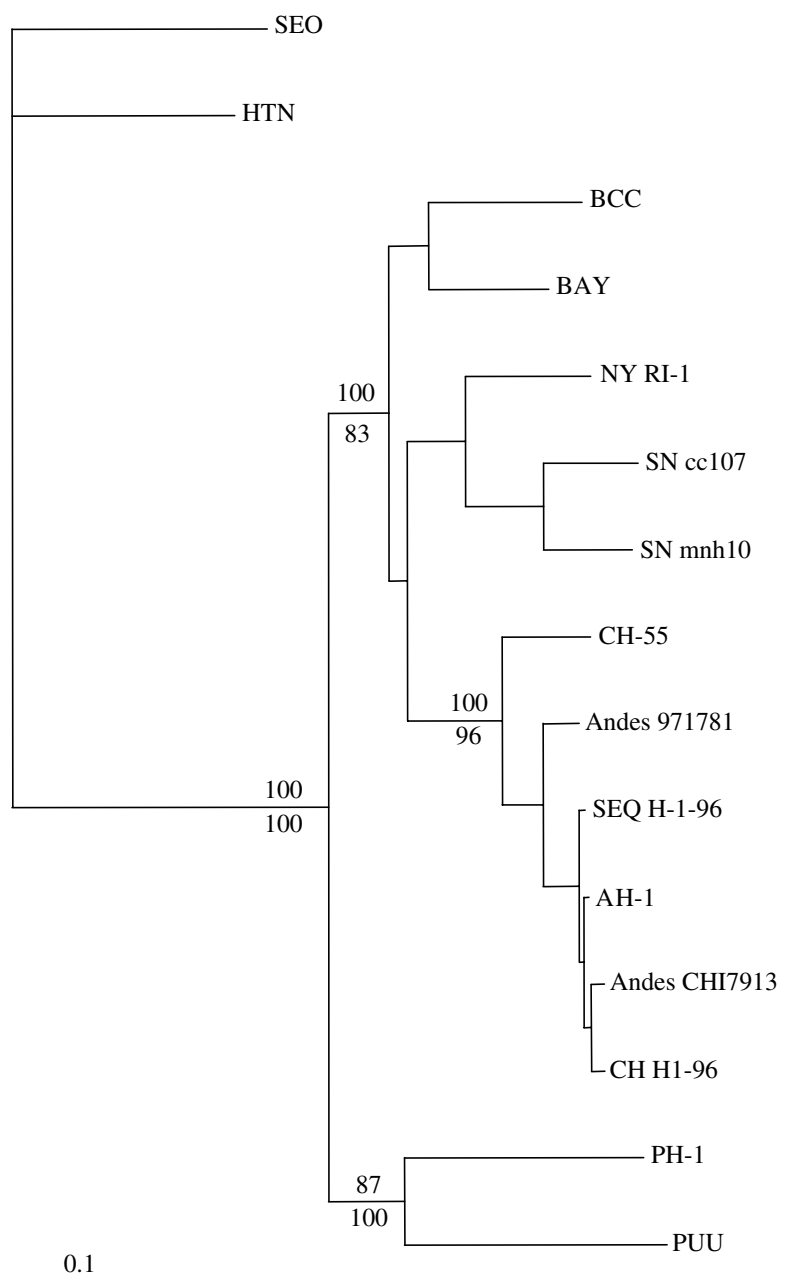

Figura 2. Análisis filogenético de virus Hanta. El árbol filogenético está basado en un segmento parcial de la nucleoproteína (nucleótido 207-511). Números de acceso al GenBank son: M34881 (SEO), M14626 (HTN), L39949 (BCC), L36929 (Bay), U09488 (NY RI-1), L33683 (SNcc107), L25784 (SN mnh1), M34011 (PH-1), X61035 (PUU), AF004660 (AH-1), AF005942 (ESQ H-1/96), AF005941 (CH H-1/96), AF291702 (CHI-7913), EU543991 (CH-55). Valores de Bootstrap obtenidos de 1000 repeticiones, los datos usando distancia y parsimonia son mostrados en el nódulo correspondiente, sólo los mayores a 50\%.

Phylogenetic analysis of Hantavirus obtained from Chile. The phylogenetic tree is based on the partial N segment (nucleotide 207-511). GenBank accession numbers for the sequences used in phylogenetic relationship are: M34881 (SEO), M14626 (HTN), L39949 (BCC), L36929 (Bay), U09488 (NY RI-1), L33683 (SNcc107), L25784 (SN mnh1), M34011 (PH-1), X61035 (PUU), AF004660 (AH-1), AF005942 (ESQ H-1/96), AF005941 (CH H-1/96), AF291702 (CHI-7913), EU543991 (CH-55) .Bootstrap values obtained from 1000 resamplings of the data using distance matrix (top) and parsimony methods (bottom) are show at the node corresponding to major lineages found in this collection of sampling, bootstrap values $>50 \%$ are shown at branching points.
A pesar de que Oligoryzomys longicaudatus es el principal reservorio para Hantavirus Andes en Chile y Argentina, existen otras especies de roedores responsables de mantener la circulación viral, aun cuando cada Hantavirus está predominantemente asociado a una sola especie como su reservorio primario natural. Sin embargo, ellos podrían simultáneamente infectar diferentes especies de roedores en una región geográfica. Por ejemplo, el virus Seoul ha sido asociado con infecciones en Rattus norvegicus y Rattus rattus en Asia (Padula y col 2002) y está ampliamente diseminado a través del mundo, aun cuando su huésped común es Rattus norvegicus (Hooper y col 1999).

El hallazgo de Hantavirus Andes en el roedor Rattus norvegicus demuestra la importancia de otros roedores como eventuales vectores virales y podría indicar que hubo una transmisión espontánea del virus de su huésped natural (Oligoryzomys longicaudatus) a otras especies de roedores con las cuales tomó contacto.

Estos resultados demostrarían la presencia de Hantavirus Andes en un roedor urbano, sin embargo desconocemos si el virus presente en este roedor es capaz de infectar a humanos.

Aun cuando hasta ahora Oligoryzomys longicaudatus ha resultado ser la especie con más alta seroprevalencia en Chile y Argentina (79\%), otras especies como Abrothrix olivaceus y Abrothrix longipilis han resultado seropositivos para Hantavirus (Castillo y col 2002 ${ }^{1}$ ). En Chile la seropositividad para Hantavirus Andes en Oligoryzomys longicaudatus es de 2,5 a 6\% entre la IV y XI Región (Castillo y col 2002) ${ }^{1}$; sin embargo, es necesario llevar a cabo estudios de seroprevalencia en un mayor número de roedores en localidades urbanas y rurales para identificar otras especies de roedores asociadas a la circulación del virus en Chile.

\section{RESUMEN}

En Chile y Argentina la especie Oligoryzomys longicaudatus ha sido identificada como el principal reservorio para Virus Andes (ANDV). El objetivo de este trabajo es reportar la presencia del virus Andes en Rattus norvergicus, roedor urbano recolectado de un muestreo en San Diego del Cristo, comuna de Melipilla, Región Metropolitana. La presencia del virus Andes en Rattus norvegicus podría indicar la importancia de otros roedores como eventuales vectores virales.

\section{REFERENCIAS}

Cantoni G, P Padula, G Calderón, J Mills, E Herrero, P Sandoval, V Martínez, N Pini, E Larrieu. 2001. Seasonal variation in prevalence of antibody to hantaviruses in rodents from southern Argentina. Trop Med Int Health 6, 811-816.

Galeno H, J Mora, E Villagra, J Fernández, J Hernández, GJ Mertz, E Ramírez. 2002. First human isolate of Hantavirus (Andes virus) in the Americas. Emerg Infect Dis 8, 657-660.

Hjelle B, T Yates. 2001. Modeling hantavirus maintenance and transmission in rodent communities. Curr Top Microbiol Immunol 256, 77-90.

Castillo C, E Palma. 2002. Actualización en Hantavirus. Archives \& Abstracts. www.medwave.cl 
Hooper JW, KI Kamrud, F Elgh, D Custer , CS Schmaljohn. 1999. DNA vaccination with hantavirus $M$ segment elicits neutralizing antibodies and protects against Seoul virus infection. Virology 15, 269-278.

Lee HW. 1999. Virus isolation. In: Manual of Hemorrhagic Fever with Renal Syndrome and Hantavirus Pulmonary Syndrome. WHO Collaborating Center for Virus Reference and Research, chapter $\mathrm{V}, \mathrm{Pp} 45$.

López N, P Padula, C Rossi, S Miguel, A Edelstein, E Ramírez. 1997. Genetic characterization and phylogeny of Andes virus and variants from Argentina and Chile. Virus Res 50, 77-84.

Mertz GJ, BL Hjelle, RT Bryan. 1997. Hantavirus infection. Adv Intern Med 42, 369-421.

Padula P, SB Colavecchia, VP Martínez, MG Della Valle, A Edelstein, SD Miguel, J Russi, JM Riquelme, N Colucci, M Almiron, RD Rabinovich. 2000. Genetic diversity, distribution, and serological features of hantavirus infection in five countries in South America. J Clin Microbiol 38, 3029-3035.
Padula P, MG Della Valle, MG Alai, P Cortada, M Villagra, A Gianella. 2002. Andes virus and first case report of Bermejo virus causing fatal pulmonary syndrome. Emerg Infect Dis 8, 437-439.

Plyusnin A, SP Morzunov. 2001. Virus evolution and genetic diversity of hantaviruses and their rodent hosts. Curr Top Microbiol Immunol 256, 47-75.

Rossi C, T Ksiazek. 1999. Virus detection and identification with serological tests. 2. Enzyme-linked immunosorbent assay (ELISA). In: Lee HW, Calisher C, Schmaljohn C (eds). Manual of hemorrhagic fever with renal syndrome and hantavirus pulmonary syndrome. WHO Collaborating Center for Virus Reference and Research (Hantavirus), Seoul, Korea, Pp 87-91.

Sotomayor V, B Marincovich. 2005. Hantavirus. Boletín de Vigilancia en Salud Pública El Vigía 9, 23.

Tager Frey M, PC Vial, CH Castillo, PM Godoy, B Hjelle, MG Ferres. 2003. Hantavirus prevalence in the IX Region of Chile. Emerg Infect Dis 9, 827-32.

Wells RM, J Young, RJ Williams, LR Armstrong, K Busico, AS Khan, TG Ksiazek, PE Rollin, SR Zaki, ST Nicho, CJ Peters. 1997. Hantavirus transmissions in the United States. Emerg Infect Dis 3, 361-5. 